\title{
Nogo-A is critical for pro-inflammatory gene regulation in myocytes and macrophages
}

\section{H M Arif Ullah}

Kyungpook National University

\section{A. K. Elfadl}

Kyungpook National University

\section{SunYoung Park}

Kyungpook National University

Yong Deuk Kim

Kyungpook National University

Myung-Jin Chung

Kyungpook National University

\section{Ji-Yoon Son}

Kyungpook National University

Hyun-Ho Yun

Kyungpook National University

Jae-Min Park

Kyungpook National University

Jae-Hyuk Yim

Kyungpook National University

Seung-Jun Jung

Kyungpook National University

Young-Chul Choi

Yonsei University College of Medicine

Jin-Hong Shin

Pusan National University Yangsan Hospital

\section{Dae-Seong Kim}

Pusan National University Yangsan Hospital

Jin-Kyu Park

Kyungpook National University

Kyu-Shik Jeong ( $\sim$ jeongks@knu.ac.kr)

Kyungpook National University 
Keywords: Nogo-A, CHOP, macrophages, pro-inflammatory factors, inflammation

Posted Date: October 2nd, 2020

DOI: https://doi.org/10.21203/rs.3.rs-84136/v1

License: (c) (1) This work is licensed under a Creative Commons Attribution 4.0 International License. Read Full License

Version of Record: A version of this preprint was published at Cells on January 31st, 2021. See the published version at https://doi.org/10.3390/cells10020282. 


\section{Abstract}

Background: Nogo-A (Rtn 4A), a member of the reticulon 4 (Rtn4) protein family, is a neurite outgrowth inhibitor protein that is primarily expressed in the central nervous system (CNS). However, the role of Nogo-A in inflammatory mechanisms remains unclear. Therefore, in this study, we used Nogo-knockout (KO) mice to explore its potential role in the inflammatory process. Here, we investigated whether Nogo-A affects the inflammatory process through transcription factor C/EBP homologous protein (CHOP).

Results: Our results demonstrated that Nogo-A, CHOP, and pro-inflammatory factors were activated in the following: notexin-induced muscle injury, in human Duchenne muscular dystrophy (DMD) patients, in dystrophin-deficient (mdx) mice, in differentiated C2C12 myoblast cells, and in lipopolysaccharide (LPS)stimulated bone marrow-derived macrophages (BMDM). Moreover, we found that Nogo-KO BMDM exhibited lower migratory ability compared with wild type (WT) BMDM after LPS treatment.

Conclusion: Our data demonstrated that the Nogo-A-CHOP signaling pathway regulated the inflammatory process in notexin-induced injured muscle, in mdx mice, in DMD patients, in differentiated C2C12 cells, and in LPS-stimulated BMDM. Taken together, these results suggest that Nogo-A plays a vital role in inflammatory processes, which resembles the pathological mechanisms observed in the CNS.

\section{Background}

Inflammation is the host's fundamental protective biological response to harmful stimuli, which include infections and tissue damage [1-3]. Dysregulated inflammatory responses contribute to the pathophysiology of many chronic diseases [1], and excessive inflammatory responses can damage muscle fibers, which can lead to muscle fibrosis, delays in tissue regeneration, and chronic muscle injury $[4,5]$. Pro-inflammatory factors are crucial factors in muscle disorders, such as Duchenne muscular dystrophy (DMD), which is a progressive form of muscular dystrophy [6]. Tissue resident macrophages play an essential role in tissue homeostasis and in the resolution of inflammation [7].

Macrophages are innate immune cells that can differentiate into different phenotypes in response to environmental cues. The two canonical types of macrophages are pro-inflammatory (M1 macrophages) and anti-inflammatory (M2 macrophages) [8, 9]. M1 macrophages secrete pro-inflammatory cytokines, chemokines, and enzymes, such as interleukin (IL)-6, IL-1 $\beta$, tumor necrosis factor (TNF)-a, nuclear factor (NF)-KB, chemokine (C-X-C motif) ligand 1 (CXCL1), chemokine (C-X-C motif) ligand 2 (CXCL2), and inducible nitric oxide synthase (iNOS), which are important for multiple inflammatory processes $[1,8,10]$. In contrast, M2 macrophages produce anti-inflammatory factors, such as IL-10, cluster of differentiation (CD)-206, and arginase (ARG)-1, which are involved in the resolution of inflammatory processes and the mediation of wound healing $[1,8]$. Due to the diverse functions of macrophages in controlling immune responses and metabolism, dysregulation of macrophage polarization is associated with disease [11]. Notexin is a myotoxic agent, and lipopolysaccharide (LPS), which is a component of bacterial endotoxin, 
is involved in severe inflammation by stimulating various pro-inflammatory factors [12-14]. However, the molecular mechanisms through which Nogo-A induces inflammation remain unknown.

As a major site of protein folding, the endoplasmic reticulum (ER) is an important cellular organelle [1517]. ER stress occurs when unfolded or misfolded proteins accumulate in the ER $[16,18]$. Three protein sensors are located at the ER membrane, where they activate transcription factor (ATF)-6, inositol requiring enzyme (IRE)-1a, and PKR-like ER kinase (PERK), which function in the identification of increased ER stress and subsequently activate the unfolded protein response (UPR) [14, 19]. Activation of the UPR results in activation and upregulation of C/EBP homologous protein (CHOP) [20,21], which is a transcription factor that indicates ER stress [22]. ER stress is an essential cellular response that triggers inflammation $[14,16,18]$. Several studies have revealed that ER stress is involved in various pathophysiological conditions including autoimmune diseases, inflammatory diseases, neurodegenerative diseases, cancer, and metabolic diseases [14, 22, 23].

Nogo is a member of the reticulon 4 (Rtn4) family of proteins, is localized within the ER membrane, and is essential for the regulation of the tubular structure of the ER [24-26]. Nogo, which has three splicing isoforms (Nogo-A (Rtn 4A), Nogo-B (Rtn 4B), and Nogo-C (Rtn 4C), is known as a neurite outgrowth inhibitor. These isoforms contain the same carboxy terminal but different amino terminals [26-28]. NogoA $(200 \mathrm{kDa})$ is a high- molecular weight membrane protein that is primarily expressed in the central nervous system (CNS). Nogo-A acts as a growth inhibitory factor [29-31] that influences the migration of cells in the neural tube and is a key negative regulator of angiogenesis in the CNS [29]. In the adult CNS, Nogo-A has also been shown to be a vital inhibitory factor of axonal regeneration and plasticity [29, 32]. Nogo-B $(55 \mathrm{kDa})$ is a shorter isoform than Nogo-A and has been shown to be expressed in cardiac myocytes and vascular cells in multiple cell types both in vitro and in vivo [33]. Expression of Nogo-B is reduced after injury in the femoral arteries of mice [24, 33]. In addition, Nogo-B regulates the migration of endothelial cells in peripheral blood vessels, which results in vascular remodeling [34, 35]. Nogo-C $(25 \mathrm{kDa})$ is the smallest protein in the Nogo family $[26,36]$ and is expressed in a variety of tissues and cells including neurons, liver cells, muscle cells, vascular smooth muscle cells, and cardiac cells [36]. Previous studies have shown that Nogo-C regulates apoptosis in cardiomyocytes during mouse myocardial infarction ( $\mathrm{Ml}$ ) and that Nogo-C deficiency improves cardiac activity after $\mathrm{MI}$ [26]. In addition, Nogo-C expression is negatively correlated with tumor size and prognosis in hepatic carcinoma [36, 37]. However, the endogenous role of Nogo-A in non-neural cells and the function of Nogo-A in inflammation are unknown.

In this study, we aimed to investigate the potential role of Nogo-A in the activation of inflammatory mediators in myocytes and macrophages. We found that Nogo knockout leads to decreased transcription of inflammation-related genes after injury. Consistently, we found that Nogo-A regulates $\mathrm{CHOP}$ and inflammatory factors in differentiated $\mathrm{C} 2 \mathrm{C} 12$ cells. In DMD models (humans and mdx mice), the expression of Nogo-A, CHOP, IL-6, and TNF- $\alpha$ is significantly upregulated. We also identified Nogo-A regulation and the CHOP pathway as a potential mechanism of inflammation. Thus, this study reveals the potential role of Nogo-A in the regulation of inflammatory mechanisms. 


\section{Results}

\section{Nogo-A, CHOP, and pro-inflammatory factors are upregulated in injured muscle}

First, we aimed to determine the role of Nogo-A in muscle inflammation. To achieve this, we used notexin, which is a myotoxic agent used to induce muscle injury $[38,39]$. We tested whether Nogo-A was activated three days after notexin-induced injury in muscle. We found that Nogo-A levels were significantly increased in notexin-injured muscle, while Nogo-B was not significantly altered in either the control or the notexin-treated muscle; however, Nogo- $\mathrm{C}$ was reduced in notexin-treated muscle compared with the control (Fig. 1a). Recent research has noted that retinal excitotoxicity results in the upregulation of NogoA expression [40]. Our results suggest that only Nogo-A, but not Nogo-B or Nogo-C, is activated in injured muscle. We also found that levels of CHOP and pro-inflammatory cytokines, such as IL- 6 and TNF-a, were also elevated in the notexin-treated mice (Fig. 1b). A previous study showed that CHOP contributes to cytokine-induced pro-inflammatory responses [41]. Using immunofluorescence (IF) analysis, we found that levels of Nogo-A, cluster of differentiation (CD)-68 (a marker of macrophages), and inducible nitric oxide synthase (iNOS) (a pro-inflammatory marker), were increased in notexin-injured muscle (Fig. 1c, d). In addition, the mRNA levels of pro-inflammatory enzymes and cytokines including iNOS, IL-1 $\beta$, and NFKB and chemokines including CXCL1 and CXCL2 were also upregulated in notexin-treated mice compared with untreated mice (Supplementary Fig. S1). Together, these results suggest that the levels of Nogo-A, $\mathrm{CHOP}$, pro-inflammatory cytokines, and chemokines are increased in notexin-induced muscle injury.

\section{Pro-inflammatory factor expression mediated by CHOP signaling is Nogo-A-dependent}

We next aimed to determine the role of Nogo-A in the regulation of the inflammatory process. To this end, we used wild type (WT) and Nogo-knockout (KO) mice and measured the levels of Nogo-A and CHOP in the muscle of mice treated with notexin. An immunoblot (IB) analysis revealed significant upregulation of Nogo-A and CHOP in WT notexin-treated muscle relative to Nogo-KO mice (Fig. 2a). Using IF, we observed higher levels of CHOP expression in WT muscle compared with Nogo-KO muscle after notexin treatment (Fig. 2b). We also found significantly higher levels of Nogo-A mRNA in notexin-treated WT muscle compared with untreated control WT muscle (Fig. 2c). CHOP, IL-6, and TNF-a mRNA levels were significantly elevated in notexin-treated WT muscle compared with notexin-treated Nogo-KO muscle, while IL-6 levels were increased in WT control mice compared with Nogo-KO control mice (Fig. 2d-f). A previous study showed that Nogo-A antibody treatment decreased the expression of inflammation-related genes [40]. These results suggest that Nogo-A upregulates $\mathrm{CHOP}$, IL-6, and TNF- $a$ expression. H\&E staining showed that inflammatory cells infiltrated in notexin treated WT and Nogo KO mice compared with control groups (Supplementary Fig. S2). To further examine the role of Nogo-A in ER stress, we administered a single dose $(1 \mu \mathrm{g} / \mathrm{kg})$ of tunicamycin, a pharmacological ER stress inducer, via intraperitoneal (IP) injection into WT and Nogo-KO mice [42]. Using PPCR, we found that the levels of Nogo-A, CHOP, IL-6, and TNF-a were significantly upregulated in WT mice relative to Nogo-KO mice (Supplementary Fig. S3A-D).

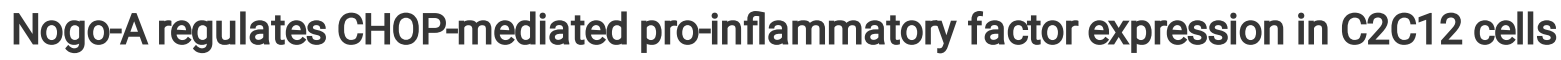


We next investigated whether Nogo-A affects the expression of pro-inflammatory factors in vitro. We evaluated the effects of Nogo-A using oligonucleotide small interfering RNA (si-Nogo-A) in C2C12 myoblast cells. We assessed differentiation-induced pro-inflammatory gene expression and found that Nogo-A knockdown led to significantly reduced levels of CHOP, IL-6, and TNF-a (Fig. 3a), as JB Mdzomba et al. showed that Nogo-A antibodies inhibit inflammation [40]. IF data also showed that Nogo-A silencing remarkably decreased Nogo-A and CHOP expression (Fig. 3b). These results indicate that Nogo-A could regulate $\mathrm{CHOP}, \mathrm{IL}-6$, and TNF-a expression in $\mathrm{C} 2 \mathrm{C} 12$ cells.

To further assess the potential role of Nogo-A, we used an adenoviral delivery system to upregulate NogoA (Ad-Nogo), after which we assessed the expression levels of Nogo-A, CHOP, IL-6, and TNF-a. The protein levels of Nogo-A and CHOP were increased in the Ad-Nogo-infected group relative to the control group (Fig. 3c). The mRNA levels of Nogo-A, CHOP, IL-6, and TNF-a were also significantly increased after infection with Ad-Nogo relative to the control (Ad-GFP) (Fig. 3d). These data suggest that Nogo-A enhances activation of the CHOP signaling pathway, which could induce pro-inflammatory gene transcription.

To examine the role of $\mathrm{CHOP}$ in the regulation of pro-inflammatory factor expression, we assessed $\mathrm{CHOP}$ overexpression using an adenoviral delivery system (Ad-CHOP) and CHOP knockdown using small interfering RNA (si-CHOP) in C2C12 cells. We demonstrated that high CHOP expression by Ad-CHOP significantly promoted IL-6 and TNF-a mRNA expression compared with the Ad-GFP (control) (Fig. 3e). In contrast, the expression of pro-inflammatory factors was dramatically reduced by $\mathrm{CHOP}$ silencing (siCHOP) (Fig. 3f), as previously described [22,43]. Moreover, LX Jia et al. reported that the mRNA levels of IL-6, IL-1 $\beta$, and CCL2 were significantly decreased in CHOP knockout mice [44]. Our results suggest that Nogo-A regulates $\mathrm{CHOP}$-mediated expression of inflammatory factors.

\section{Nogo deficiency suppresses expression of pro-inflammatory factors in BMDM}

We investigated the critical role of Nogo-A in inflammation in bone marrow-derived macrophages (BMDM). We isolated BMDM from WT and Nogo-KO mice and cultured them for seven days (Supplementary Fig. S4A). IF staining for the macrophage marker CD68 indicated that the isolated cells are macrophages (Supplementary Fig. S4B). Using qPCR, we found that Nogo-A and Nogo-C, but not Nogo-B, were significantly activated in BMDM that were treated with LPS (Fig. 4a). Using western blot, we observed that Nogo-A levels were significantly elevated in LPS-stimulated BMDM compared with control (unstimulated) BMDM (Fig. 4b). We next determined whether Nogo-A regulates the expression of proinflammatory factors in BMDM from WT and Nogo-KO mice. We found that LPS-treated Nogo-KO BMDM expressed significantly decreased levels of IL-6, TNF-a, IL-1 $\beta$, NF-KB, CXCL1, and CXCL2 compared with WT BMDM, whereas iNOS was not significantly downregulated in LPS-treated Nogo-KO BMDM compared with WT BMDM (Fig. 4c-i), as previously described [40,45]. These results suggest that Nogo-A may be involved in the activation of pro-inflammatory factors in LPS-treated WT BMDM. In contrast, the expression levels of anti-inflammatory (M2) factors, including arginase-1, CD206, and IL-10, were not significantly elevated after LPS treatment (Supplementary Fig. S5A-C). 
We next determined the role of Nogo-A in BMDM activation using the strong M2 inducer IL-4. IF staining showed that Nogo-A was expressed in control WT BMDM but that CD206 was not expressed in control BMDM derived from WT and Nogo-KO mice (Supplementary Fig. S5D). In the IL-4-treated group, CD206 expression was slightly upregulated in Nogo-KO BMDM compared with WT BMDM (Supplementary Fig. S5E). In addition, flow cytometry showed that, after IL-4 treatment, CD206 expression was similarly elevated in Nogo-KO BMDM compared with WT BMDM, but this difference was not significant (Supplementary Fig. S5F).

\section{Nogo deficiency suppresses CHOP signaling and migration of BMDM}

To develop a better understanding of the molecular relationship between Nogo-A and CHOP, we performed IF staining and found that Nogo-A was expressed in control WT BMDM and that CHOP was not expressed in either control WT or Nogo-KO BMDM (Fig. 5a). However, when Nogo-KO BMDM were treated with LPS, CHOP expression was downregulated relative to that in WT BMDM (Fig. 5b). In addition, we used qPCR to measure the levels of CHOP in control BMDM and in LPS-treated BMDM. In LPS-treated Nogo-KO BMDM, CHOP expression was significantly reduced (Fig. 5c). Moreover, IF results demonstrated that Nogo-A was co-localized with calnexin, an ER marker (Supplementary Fig. S6A, B). These results suggest that CHOP expression is reduced in Nogo-KO BMDM.

We next assessed whether Nogo-A affects the inflammatory response through the inflammation inducer LPS, as determined by the migration activity of macrophages. Interestingly, the migration assay indicated that the migration activity of BMDM was significantly decreased in LPS-treated Nogo-KO BMDM relative to WT BMDM (Fig. 5d, e). In contrast, BMDM migration did not significantly differ between the control WT and the Nogo-KO BMDM. Phagocytic activity is a fundamental biological mechanism of macrophages. Phagocytosis with zymosan is a popular technique used by macrophages [46]. To assess the involvement of the phagocytic activity of macrophages, we used an inducer of pro-inflammatory factors (zymosan) along with Alexa Fluor 488-labeleda fluorescent bioparticles. Using FACS, we found that phagocytic activity was higher in WT BMDM compared with Nogo-KO BMDM, although the difference was not statistically significant (Fig. 5f). These data suggest that Nogo deficiency prevents the migration of BMDM derived from LPS-stimulated Nogo-KO mice.

\section{Expression of Nogo-A, CHOP, and pro-inflammatory factors is increased in mdx mice and human DMD samples}

We assessed the activation of Nogo-A and CHOP in a DMD mouse model (mdx mice). Western blot showed that Nogo-A and CHOP protein levels were dramatically upregulated in mdx mice (Fig. 6a). In mdx mice, we found that the mRNA levels of Nogo-A, CHOP, IL-6, and TNF-a were also significantly increased compared with those in WT mice (Fig. 6b), as previously reported $[47,48]$. Inflammatory mediators have also been shown to participate in fibrosis in $\mathrm{mdx}$ mice [49]. These data suggest that Nogo-A, CHOP, IL-6, and TNF-a expression is increased in mdx mice compared with WT mice. 
To verify the clinical relevance of higher expression of Nogo-A, CHOP, and pro-inflammatory genes in DMD patients, we performed an immunoblot analysis and qPCR. Using western blot, we observed significantly increased levels of Nogo-A and CHOP in DMD patient samples compared with healthy donors (Fig. 6c). Finally, we found that DMD patient samples had significantly elevated levels of Nogo-A, CHOP, IL-6, and TNF-a mRNA compared with healthy donors (Fig. 6d). Taken together, these data suggest that Nogo-A promotes inflammation in $\mathrm{mdx}$ mice and DMD patients.

\section{Discussion}

Here, we discussed our understanding of the inflammatory mechanisms of Nogo-A in different models. Based on our results, we summarized the regulation of Nogo-A in inflammation in Fig. 6e. Previous studies have shown that Nogo-A is a negative regulator of axonal regeneration and that it acts as a neurite outgrowth inhibitor in the CNS [50-52]. Nogo-A levels are altered during physiological processes, such as cerebral ischemia, denervation in the CNS, brain injuries, tumors, and skeletal muscle injury [5355]. In the present study, we found that Nogo-A was elevated in muscle from notexin-treated mice, mdx mice, DMD human samples, differentiated $\mathrm{C} 2 \mathrm{C} 12$ cells, and in LPS-stimulated BMDM, whereas the levels of Nogo-B and Nogo-C were not significantly increased in notexin-treated muscle. However, different stimuli may result in different outcomes of inflammation in vivo and in vitro. Collectively, our results revealed that the upregulation of Nogo-A may play a critical role in the regulation of inflammation.

Pro-inflammatory macrophages release greater levels of chemical substances including pro-inflammatory cytokines, enzymes, and chemokines such as IL-6, IL-1 $\beta$, TNF- $a$, iNOS, CXCL1, and CXCL2 [8, 56]. TNF- $a$ is mainly induced by activation of M1 macrophages and can lead to the expression of other cytokines by M1 macrophages, including IL-6, and can regulate the inflammatory process $[57,58]$. IL-1 $\beta$ is also a vital pro-inflammatory factor that is produced by M1 macrophages and promotes activation of the NF-KB pathway $[58,59]$. In contrast, anti-inflammatory macrophages, which are also known as alternativeactivated macrophages, express higher levels of anti-inflammatory mediators, such as IL-10, CD206, and arginase-1 $[8,60]$. M2 macrophages inhibit pro-inflammatory mediators as well as their responses and promote angiogenesis and the resolution of inflammation, which are key aspects of tissue repair [61, 62].

$\mathrm{CHOP}$, a marker of ER stress, is a vital transcription factor that is induced in response to ER stress [14, 22]. We have found that Nogo-A is critical for the maintenance of inflammation but that loss of the Nogo isoforms does not lead to any apparent dysfunction under normal conditions. However, in conditions of disease or stress, we found that Nogo-A plays a role in the inflammatory cascade. We found that Nogo-A, $\mathrm{CHOP}$, and pro-inflammatory factors were increased in WT mice three days after notexin treatment. Previous work has shown that cytokines induce ER stress in vitro, as observed by increased levels of CHOP [63]. Similarly, our results suggest that upregulation of IL-6 and TNF-a in injured muscle is associated with CHOP induction and may partially involve Nogo-A in the inflammatory process.

Our current work demonstrated that Nogo-A knockdown (si-Nogo-A) in differentiated C2C12 cells caused a reduction in CHOP, IL- 6 and, TNF-a expression at the mRNA level. A previous study also stated that 
Nogo-A silencing led to the downregulation of IL-6 and TNF-a in LPS-stimulated PC12 cells [45]. IF data showed substantially decreased Nogo-A and CHOP expression after Nogo-A was silenced. Adenovirusbased overexpression of Nogo-A (Ad-Nogo) led to the increased mRNA expression of CHOP, IL-6, and TNFa in differentiated $\mathrm{C} 2 \mathrm{C} 12$ cells. Protein levels of Nogo-A and CHOP were elevated in the Ad-Nogo-infected group. Additionally, adenovirus-based overexpression of CHOP (Ad-CHOP) in $\mathrm{C} 2 \mathrm{C} 12$ cells led to increased mRNA levels of IL-6 and TNF-a, as previously reported [64]. In contrast, CHOP silencing (si-CHOP) dramatically reduced the expression of IL- 6 and TNF- $a$. Previous studies have shown that the inflammatory response was significantly reduced in CHOP knockout mice $[22,43,44]$. These results indicate that $\mathrm{CHOP}$ regulates the expression of ER stress-induced pro-inflammatory cytokines.

To establish the role of Nogo-A in this process, we isolated BMDM from WT and Nogo-KO mice. LPStreated WT BMDM exhibited significant upregulation of Nogo-A and Nogo-C but not Nogo-B compared with control WT BMDM. Our study suggested that, in contrast to Nogo-C, Nogo-A was primarily expressed in muscle fibers. Nogo-C elevation in macrophages could be due to apoptosis, as previously described [36]. However, further investigation is needed to evaluate the role of Nogo-C in inflammation. In addition, the expression levels of CHOP and pro-inflammatory factors were decreased in Nogo-KO BMDM compared with WT BMDM after LPS treatment. Other studies have reported that $\mathrm{CHOP}$ induction in response to ER stress may play a critical role in LPS-mediated inflammation [14, 16, 18, 22]. Based on our results, Nogo-A was upregulated in LPS-treated WT BMDM, which could contribute to inflammation by increasing the expression of CHOP and pro-inflammatory factors. These findings indicate that Nogo-A promotes the activation of pro-inflammatory genes and the stimulation of BMDM after LPS treatment. For the first time, our data show that migration activity of LPS-treated WT BMDM is significantly higher than that of Nogo-KO BMDM. Previous research has shown that cytokine secretion facilitates macrophage migration [65]. Therefore, Nogo-A may affect the migration of macrophages in LPSstimulated BMDM. We hypothesized that an increased number of pro-inflammatory macrophages after stimulation of WT BMDM by LPS may be linked to increased phagocytosis. Moreover, our phagocytosis assay revealed a higher trend of phagocytic activity in WT BMDM compared with Nogo-KO BMDM. Taken together, our data suggest that Nogo-A may regulate the migration and phagocytic activity of macrophages in LPS-stimulated BMDM.

Investigation of the skeletal muscle of mdx mice showed increases in Nogo-A, CHOP, IL-6, and TNF- $\mathrm{a}$ compared with WT mice. Immunoblotting revealed increased expression of Nogo-A and CHOP proteins in mdx mice compared with WT mice. Furthermore, muscle biopsies from DMD patients had significant increases in Nogo-A, CHOP, IL-6, and TNF-a expression at both the mRNA and protein levels. In mdx mice and DMD patients, elevated levels of pro-inflammatory cytokines were observed in the blood at presymptomatic stages of the disease $[12,47,48,66]$. Thus, the release of specific pro-inflammatory cytokines may stimulate the production of reactive oxygen species (ROS), which would enhance cellular damage in DMD [12]. Our findings strongly suggest a relationship between Nogo-A and pro-inflammatory cytokines in both mdx mice and DMD patients. However, an investigation of Nogo-A in muscle inflammation was not performed. 


\section{Conclusion}

In summary, we found that Nogo-A was upregulated in a notexin-induced muscle injury model, in mdx mice, in DMD human samples, in differentiated C2C12 cells, and in LPS-stimulated BMDM. The expression of pro-inflammatory factors was downregulated in Nogo-KO mice. Interestingly, Nogo-A silencing in differentiated $\mathrm{C} 2 \mathrm{C} 12$ cells led to reduced expression of CHOP, IL-6, and TNF-a. Although many inflammatory signaling pathways are involved in myopathy, we show here that Nogo-A plays a critical role in the activation of pro-inflammatory factors after inflammatory stimulation. Our findings indicate that Nogo-A might be a potential therapeutic target for the treatment of inflammatory diseases, such as myopathies, but more studies are needed to better understand the mechanisms of Nogo-A in inflammatory diseases.

\section{Materials And Methods}

\section{Muscle injury}

Muscle injury was induced by a single intramuscular (IM) injection of $20 \mu \mathrm{l}$ of the myotoxic agent notexin $(12.5 \mu \mathrm{g} / \mathrm{ml}$, Latoxan, Valence, France), diluted in PBS, (or with $20 \mu \mathrm{l} \mathrm{PBS}$ as a control) into the gastrocnemius muscle of experimental mice. Briefly, WT and Nogo-KO mice (3 mice per group) were anesthetized after which both hind limbs were shaved. Notexin was injected into the right leg muscle, while the muscle of the left leg served as a control and was injected with PBS. Three days after notexin injection, the mice were euthanized and the gastrocnemius muscle was surgically isolated, as previously described $[12,67]$. The gastrocnemius muscle was cut in half as a cross-section, fixed in $4 \%$ paraformaldehyde (PFA) overnight, and subsequently transferred to $30 \%$ sucrose in PBS for 24 hours. Using optimum cutting temperature (OCT) medium, the samples were embedded in a cryo block for histological analysis. The remaining half of the muscle sample was immediately frozen in liquid nitrogen for molecular analysis. The sample was subsequently stored at $-80^{\circ} \mathrm{C}$ until further analysis. All animal studies were approved by the Institutional Animal Use and Care Committee of Kyungpook National University, Daegu, Republic of Korea (KNU 2017-0023 and 2018-0074).

\section{Mouse model of Duchenne muscular dystrophy}

Mice were housed under the appropriate conditions with 12-hour light cycles and were supplied with water and food in accordance with the regulations of the Kyungpook National University animal facility. For the DMD animal model, male WT mice (C57BL/6J, 12 weeks of age, $n=10$ ) and male mdx mice (C57BL/10ScSn-Dmdmdx/J, 12 weeks of age, $n=4$ ) were used. Mdx mice were a gift from Jacques Tremblay (CHUQ Research Center, Quebec City, Canada), and C57BL/6J mice were purchased from Japan SLC, Incorporated. (Hamamatsu, Japan), as previously described [68].

\section{Induction of endoplasmic reticulum (ER) stress using tunicamycin}


Healthy ten-week-old mice were housed under the appropriate conditions and were divided into the following 4 groups: WT mice without tunicamycin treatment $(n=3)$, WT mice treated with tunicamycin ( $n$ $=4)$, Nogo-KO mice without tunicamycin treatment $(\mathrm{n}=4)$, and Nogo-KO mice treated with tunicamycin ( $\mathrm{n}$ =5). Tunicamycin was administered at a single dose of $(1 \mu \mathrm{g} / \mathrm{kg})$ via intraperitoneal (IP) injection.

Muscles were harvested 24 hours after injection. Tunicamycin was prepared in DMSO and diluted in PBS to reduce the toxicity of DMSO in mice.

\section{Human myopathy}

Muscle biopsies were obtained from patients at the Pusan National University Yangsan Hospital (Yangsan, Republic of Korea, Institutional review board No. 05-2018-045) and Yonsei University College of Medicine (Seoul, Republic of Korea, Institutional review board No. 3-2018-0060) after approval from the Medical School Ethical Committee. Muscle samples were harvested after diagnosis, and informed consent was obtained from all patients for the scientific use of their muscle biopsy specimens. The samples from patients with myopathy and those with DMD were collected according to the patient's age (22 months, 2 years, 5 years, 5 years 1 month, 5 years 7 months, 15 years, 20 years, 46 years, 57 years, and 81 years). Four muscle biopsies were obtained for each group from age-matched healthy control patients (18 years, 26 years, 41 years, and 42 years). qPCR and immunoblot analyses were performed on all muscle biopsy samples.

\section{C2C12 cell culture}

The murine myoblast cell line (C2C12) was cultured in Dulbecco's Modified Eagle's Medium (DMEM; Gibco-BRL, Grand Island, NY, USA) supplemented with 10\% fetal bovine serum (FBS; Hyclone, Logan, UT, USA) and $1 \%$ penicillin/streptomycin (P/S). Cells were cultured in a humidified incubator containing $5 \%$ $\mathrm{CO}_{2}$ at $37^{\circ} \mathrm{C}$. C2C12 cells were grown until they were $60 \%-70 \%$ confluent. Cells were then sub-cultured and grown for another 48 hours. Finally, the cells were differentiated in $2 \%$ horse serum for 3 days, as previously described $[69,70]$.

\section{Recombinant adenovirus and si-RNA transfection of C2C12 cells}

Ad-Nogo-A and Ad-CHOP were purchased from Vector Biolabs (Malvern, PA, USA). The small interfering (si) RNAs against Nogo-A and CHOP (si-cram, si-Nogo-A, and si-CHOP) were purchased from Bioneer Research (Seoul, Republic of Korea) and were transfected into cells using Lipofectamine 2000 reagent (Invitrogen, Carlsbad, CA, USA) according to the manufacturer's instructions. For transfections, the cells were plated in 60-mm dishes at a density of $1 \times 10^{5}$ cells in DMEM without antibiotics and allowed to grow for 24 hours. When the cells became $40 \%-50 \%$ confluent, the cells were transfected according to the manufacturer's instructions.

\section{Isolation, culture, and activation of bone marrow-derived macrophages}


Macrophages were obtained from bone marrow with several modifications, as previously described [7, 71]. Briefly, bone marrow cells were obtained by flushing the femur and tibia of 8-week-old C57BL/6 (WT) and Nogo-knockout $(\mathrm{KO})$ mice $(\mathrm{n}=6)$. The femur and tibia were washed with $70 \%$ ethanol and then with PBS. Sterile scissors were used to cut both the knee and hip joints. The ends of the femur and tibia bones were also cut to obtain macrophages from the bone marrow. The bone marrow was flushed out in a 50$\mathrm{ml}$ Falcon tube using a 26 gauge syringe and sterile PBS. The sample was then centrifuged at $3,000 \mathrm{~g}$ for 5 minutes at $4{ }^{\circ} \mathrm{C}$ after which the cells were suspended in RPMI 1640 medium containing $15 \%$ conditioned medium from the $\mathrm{L} 929$ cell line as a source of macrophage colony stimulating factor (MCSF). Cells were incubated for seven days and treated with lipopolysaccharide (LPS), an M1 inducer (100 $\mathrm{ng} / \mathrm{ml})$, or IL-4, an M2 inducer ( $20 \mathrm{ng} / \mathrm{ml}$ ) for 24 hours.

\section{Quantitative real-time polymerase chain reaction (qRT-PCR) analysis}

Total RNA was extracted from the gastrocnemius muscles of mice and from BMDM using TRIzol (Invitrogen, Carlsbad, CA, USA) according to the manufacturer's instructions. Gene expression was measured by quantitative real-time polymerase chain reaction (qPCR) using SYBR Green with low ROX (Enzynomics, catalog no. RT500S) [72, 73]. Relative quantification of the target gene was determined by normalizing expression to that of the housekeeping gene GAPDH, which served as a control. The primer sequences used in this study are listed in Table S1. qPCR data were analyzed using a CFX Connect RealTime System (Bio-Rad).

\section{Western blot analysis}

Proteins were isolated from BMDM and analyzed by immunoblotting, as previously described [74]. Briefly, the protein concentration in the samples was measured, samples were prepared in SDS and sample loading buffer, and heated for 10 minutes at $95^{\circ} \mathrm{C}$. Proteins were separated using $10 \%$ SDS-PAGE and immunoblotted onto membranes. The membranes were blocked with $1 \%$ bovine serum albumin (BSA) for 1 hour and incubated with primary antibodies, including those against Nogo-A (Abcam, catalog no. ab62024), CHOP (Santa Cruz, catalog no. sc-71136), $\beta$-actin (Cell Signaling Technology, catalog no. 8457s), and GAPDH (Cell Signaling Technology, catalog no. 2118), overnight at $4{ }^{\circ} \mathrm{C}$. After a 1-hour incubation with HRP-labeled secondary antibodies (Anti-rabbit-HRP, Cell Signaling Technology, catalog no. 7074s and Anti-mouse-HRP, Cell signaling Technology, catalog no. 7076s), the proteins were detected using enhanced chemiluminescence (ECL, SuperSignal West Dura Extended Duration Substrate, catalog no. 34076) in an Amersham Imager 680 (GE Healthcare, Life Sciences). Blots were quantified using ImageJ software.

\section{Immunofluorescence (IF) assay}

The immunofluorescence assay was performed with modifications, as previously described [75]. Briefly, cryosections and BMDM were washed with tris-buffered saline (TBS) and fixed in $4 \%$ paraformaldehyde (PFA) for 10 minutes. After washing, the samples were permeabilized with TBST $(0.2 \%$ Triton X-100 in TBS) for 10 minutes and washed three times with TBS for 5 minutes. Samples were blocked using 2\% 
BSA after which the cells were incubated with primary antibodies, including rabbit anti-Nogo-A (Abcam, catalog no. ab62024), mouse anti-CD68 (Santa Cruz Biotechnology, catalog no. ab955), mouse anti-iNOS (Santa Cruz Biotechnology, ab49999), mouse anti-CD206 (Santa Cruz Biotechnology, catalog no. sc58986), mouse anti-CHOP (Santa Cruz Biotechnology, sc-71136), and mouse anti-calnexin (Novus Biologicals, catalog no. NB300518), at $4{ }^{\circ} \mathrm{C}$ overnight. The cells were then washed 3 times with TBS for 5 minutes each wash. Samples were incubated with secondary antibodies (donkey anti-mouse immunoglobulins (Alexa Fluor 488, Abcam, catalog no. ab150105) and donkey anti-rabbit immunoglobulins (Alexa Fluor 555, Abcam, catalog no. ab150066) for 1 hour in the dark. The samples were mounted using ProLong ${ }^{\text {TM }}$ Gold Antifade reagent containing DAPI to visualize the nuclei (Cell Signaling Technology, catalog no. 8961s) and were analyzed by confocal microscopy (ZEISS).

\section{Histological analysis}

Gastrocnemius muscles from mice samples were rapidly fixed with $5 \%$ sucrose in $4 \%$ paraformaldehyde (PFA) for 24 hours and subsequently transferred into $30 \%$ sucrose in PBS for 24 hours. Samples were embedded in OCT compound for cryopreservation. Cryosections of $5 \mu \mathrm{m}$ thick tissues were cut for histological analysis. Sections of muscle were stained with hematoxylin and eosin (H\&E). Stained tissue sections were visualized using a light microscope.

\section{Flow cytometry analysis}

BMDM were incubated for seven days and stimulated with lipopolysaccharide (LPS) $(100 \mathrm{ng} / \mathrm{ml})$ or IL-4 $(20 \mathrm{ng} / \mathrm{ml})$ for 24 hours. BMDM were collected and washed twice in PBS and centrifuged at $1500 \mathrm{~g}$ for 3 minutes. Cells were incubated at $37^{\circ} \mathrm{C}$ with primary antibodies against iNOS (Abcam, catalog no. ab49999) and CD206 (santa cruz, catalog no. sc-58986) for 1 hour and were then washed with PBS. Finally, the cells were incubated with fluorochrome-labeled secondary antibodies in PBS for 30 minutes. After three washes in PBS, the cells were analyzed by flow cytometry.

\section{Migration assay}

A migration assay was performed with modifications, as previously described [76]. Transwell chambers (6.5 mm diameter and $8 \mu \mathrm{m}$ pore size) were obtained from Corning (catalog no. 3422). BMDM were harvested and suspended in RPMI supplemented with $10 \%$ FBS at a concentration of $2 \times 10^{4}$ cells/well. Cells were seeded in serum-free medium into the upper chamber of a 24-well plate. The lower chambers were filled with RPMI medium containing $10 \%$ FBS. Cells were incubated overnight. Cells that had migrated to the reverse side of the Transwell membrane were fixed in 4\% PFA and permeabilized with absolute methanol. Cells were stained with $\mathrm{H} \& \mathrm{E}$, and non-migrated cells were removed using cotton swabs at which point the cells that had migrated were counted using a light microscope.

\section{Phagocytosis assay}


BMDM were stimulated with Alexa Fluor 488-labeled zymosan fluorescent bioparticles (catalog no. z23373). For flow cytometry, the BMDM were washed twice in PBS. Adherent cells were detached as a result of incubation with trypsin-EDTA for 5 minutes in the incubator and were subsequently centrifuged at $1500 \mathrm{~g}$ for 3 minutes. Cells were placed in the incubator and given 30 minutes to internalize the zymosan particles. Noninternalized particles were removed by three washes in cold PBS. The harvested cells were then washed and fixed in $4 \%$ paraformaldehyde. Cells were washed twice with PBS, placed in a FACS tube, and were immediately examined by flow cytometry.

\section{Statistical analysis}

Statistical analysis was performed using GraphPad Prism 6.01 (GraphPad Software) program. Statistical significance was determined using Student's t-test. Data are expressed as means and standard error of the mean (SEM). The statistical significance of data is denoted on the graphs by asterisks $\left({ }^{*}\right)$, with $P$ values of $* P<0.05,{ }^{*} P<0.01$, and ${ }^{* \star *} P<0.001$

\section{Abbreviations}

Rtn4: Reticulon 4; KO: knockout; CNS: central nervous system; WT: wild type; CHOP: C/EBP homologous protein; IL-6: Interleukin-6; TNF-a: tumor necrosis factor-a; NF-kB: nuclear factor-kB; DMD: Duchenne muscular dystrophy; BMDM: bone marrow-derived macrophages; ER: endoplasmic reticulum; LPS: lipopolysaccharide; TBS: tris-buffered saline; PFA: paraformaldehyde.

\section{Declarations}

\section{Acknowledgments}

Not applicable.

\section{Authors' contributions}

Conceptualization: HMAU, AKE, SP, MJC, JYS, HHY, JMP, JHY, SJJ, YCC, JHS, DSK, JKP and JKS; Validation: HMAU, AKE, SP and JKS; Performed experiments and formal analysis: HMAU and AKE; Investigation: JKS; Writing-original draft: HMAU, AKE and JKS; Writing-review and editing: HMAU, SP and JKS; Visualization: HMAU, AKE, SP and JKS; Supervision: JKS; Funding Acquisition: JKS. All authors read and approved the final manuscript.

\section{Funding}

This work was financially supported by the government of Republic of Korea (Ministry of Science and ICT) (NRF-2017R1E1A1A01072781).

\section{Availability of data and materials}


All data generated or analyzed during this study are included in this published article (and its supplementary information files).

\section{Ethics approval and consent participate}

Human muscle samples with informed consent were obtained from healthy volunteers and patients at the Pusan National University Yangsan Hospital, Yangsan, Republic of Korea, and Yonsei University College of Medicine, Seoul, Republic of Korea after approval from the Medical School Ethical Committee. The animal studies were approved by the Institutional Animal Use and Care Committee of Kyungpook National University, Daegu, Republic of Korea.

\section{Consent for publication}

Not applicable.

\section{Competing interests}

The authors have no potential conflict of interests to disclose.

\section{References}

1. Liu T, Zhang L, Joo D, Sun S-C. NF-kB signaling in inflammation. Signal Transduct Target Ther. 2017;2(1):1-9.

2. Zhang H, Sun S-C. NF-KB in inflammation and renal diseases. Cell Biosci. 2015;5(1):63.

3. Lawrence T. The nuclear factor NF-KB pathway in inflammation. Cold Spring Harb Perspect Biol. 2009;1(6):a001651.

4. Porter JD, Khanna S, Kaminski HJ, Rao JS, Merriam AP, Richmonds CR, et al. A chronic inflammatory response dominates the skeletal muscle molecular signature in dystrophin-deficient mdx mice. Hum Mol Genet. 2002;11(3):263-72.

5. Liu X, Wu G, Shi D, Zhu R, Zeng H, Cao B, et al. Effects of nitric oxide on notexin-induced muscle inflammatory responses. Int J Biol Sci. 2015;11(2):156.

6. Chung HY, Sung B, Jung KJ, Zou Y, Yu BP. The molecular inflammatory process in aging. Antioxid Redox Sign. 2006;8(3-4):572-81.

7. Arnold L, Perrin H, De Chanville CB, Saclier M, Hermand P, Poupel L, et al. CX3CR1 deficiency promotes muscle repair and regeneration by enhancing macrophage ApoE production. Nat. Commun. 2015;6:8972.

8. Park JK, Shao M, Kim MY, Baik SK, Cho MY, Utsumi T, et al. An endoplasmic reticulum protein, Nogo$B$, facilitates alcoholic liver disease through regulation of kupffer cell polarization. Hepatol. 2017;65(5):1720-34.

9. Wynn TA, Chawla A, Pollard JW. Macrophage biology in development, homeostasis and disease. Nature. 2013;496(7446):445-55. 
10. Biswas SK, Mantovani A. Orchestration of metabolism by macrophages. Cell Metab. 2012;15(4):4327.

11. Schultze JL, Schmieder A, Goerdt S, editors. Macrophage activation in human diseases. Semin Immunol. 2015: Elsevier.

12. Park M, Lee B-S, Jeon S-H, Nam H-J, Lee G, Kim C-H, et al. A novel isoform of met receptor tyrosine kinase blocks hepatocyte growth factor/Met signaling and stimulates skeletal muscle cell differentiation. J Biol Chem. 2015;290(3):1804-17.

13. Schertzer J, Lynch G. Comparative evaluation of IGF-I gene transfer and IGF-I protein administration for enhancing skeletal muscle regeneration after injury. Gene Ther. 2006;13(23):1657.

14. Kim HJ, Jeong JS, Kim SR, Park SY, Chae HJ, Lee YC. Inhibition of endoplasmic reticulum stress alleviates lipopolysaccharide-induced lung inflammation through modulation of NF-KB/HIF-1a signaling pathway. Sci Rep. 2013;3(1):1-10.

15. Ron D, Walter P. Signal integration in the endoplasmic reticulum unfolded protein response. Nat Rev Mol Cell Biol. 2007;8(7):519-29.

16. Hotamisligil GS. Endoplasmic reticulum stress and the inflammatory basis of metabolic disease. Cell. 2010;140(6):900-17.

17. Filipe A, Chernorudskiy A, Arbogast S, Varone E, Villar-Quiles R-N, Pozzer D, et al. Defective endoplasmic reticulum-mitochondria contacts and bioenergetics in SEPN1- related myopathy. Cell Death Differ. 2020:1-16.

18. Zhang K, Kaufman RJ. From endoplasmic-reticulum stress to the inflammatory response. Nature. 2008;454(7203):455-62.

19. Oyadomari S, Mori M. Roles of CHOP/GADD153 in endoplasmic reticulum stress. Cell Death Differ. 2004;11(4):381-9.

20. Gorman AM, Healy SJ, Jäger R, Samali A. Stress management at the ER: regulators of ER stressinduced apoptosis. Pharmacol Therapeut. 2012;134(3):306-16.

21. Liu Z, Shi Q, Song X, Wang Y, Wang Y, Song E, et al. Activating transcription factor 4 (ATF4)-ATF3$\mathrm{C} / \mathrm{EBP}$ homologous protein (CHOP) cascade shows an essential role in the ER stress-induced sensitization of tetrachlorobenzoquinone-challenged PC12 cells to ROS-mediated apoptosis via death receptor 5 (DR5) signaling. Chem Res Toxicol. 2016;29(9):1510-8.

22. Endo $M$, Mori M, Akira S, Gotoh T. C/EBP Homologous Protein (CHOP) Is Crucial for the Induction of Caspase-11 and the Pathogenesis of Lipopolysaccharide-Induced Inflammatio. J Immunol. 2006;176(10):6245-53.

23. Sano R, Reed JC. ER stress-induced cell death mechanisms. BBA-Mol Cell Res. 2013;1833(12):346070.

24. Sutendra G, Dromparis P, Wright P, Bonnet S, Haromy A, Hao Z, et al. The role of Nogo and the mitochondria-endoplasmic reticulum unit in pulmonary hypertension. Sci Transl Med. 2011;3(88):88ra55-88ra55. 
25. Voeltz GK, Prinz WA, Shibata Y, Rist JM, Rapoport TA. A class of membrane proteins shaping the tubular endoplasmic reticulum. Cell. 2006;124(3):573-86.

26. Weng L, Jia S, Xu C, Ye J, Cao Y, Liu Y, et al. Nogo-C regulates post myocardial infarction fibrosis through the interaction with ER Ca 2+ leakage channel Sec61a in mouse hearts. Cell Death Dis. 2018;9(6):1-15.

27. Dodd DA, Niederoest B, Bloechlinger S, Dupuis L, Loeffler J-P, Schwab ME. Nogo-A,-B, and-C are found on the cell surface and interact together in many different cell types. J Biol Chem. 2005;280(13):12494-502.

28. Oertle T, Klinger M, Stuermer CA, Schwab ME. A reticular rhapsody: phylogenic evolution and nomenclature of the RTN/Nogo gene family. FASEB J. 2003;17(10):1238-47.

29. Wälchli T, Pernet V, Weinmann O, Shiu J-Y, Guzik-Kornacka A, Decrey G, et al. Nogo-A is a negative regulator of CNS angiogenesis. Proc Natl Acad Sci. 2013;110(21):E1943-E52.

30. Schwab ME. Nogo and axon regeneration. Curr Opin Neurobiol. 2004;14(1):118-24.

31. Chen MS, Huber AB, van der Haar ME, Frank M, Schnell L, Spillmann AA, et al. Nogo-A is a myelinassociated neurite outgrowth inhibitor and an antigen for monoclonal antibody IN-1. Nature. 2000;403(6768):434-9.

32. Schwab ME. Functions of Nogo proteins and their receptors in the nervous system. Nat Rev Neurosci. 2010;11(12):799-811.

33. Huber AB, Weinmann O, Brösamle C, Oertle T, Schwab ME. Patterns of Nogo mRNA and protein expression in the developing and adult rat and after CNS lesions. J Neurosci. 2002;22(9):3553-67.

34. Acevedo L, Yu J, Erdjument-Bromage H, Miao RQ, Kim J-E, Fulton D, et al. A new role for Nogo as a regulator of vascular remodeling. Nat Med. 2004;10(4):382-8.

35. Kritz AB, Yu J, Wright PL, Wan S, George SJ, Halliday C, et al. In vivo modulation of Nogo-B attenuates neointima formation. Mol Thera. 2008;16(11):1798-804.

36. Jia S, Qiao X, Ye J, Fang X, Xu C, Cao Y, et al. Nogo-C regulates cardiomyocyte apoptosis during mouse myocardial infarction. Cell Death Dis. 2016;7(10):e2432-e.

37. Chen $Y$, Tang $X$, Zhang X, Zhuang L. New mutations of Nogo-C in hepatocellular carcinoma. Mol Biol Rep. 2009;36(2):377-80.

38. Hardy D, Fefeu M, Besnard A, Briand D, Gasse P, Arenzana-Seisdedos F, et al. Defective angiogenesis in CXCL12 mutant mice impairs skeletal muscle regeneration. Skeletal muscle. 2019;9(1):25.

39. Hardy D, Besnard A, Latil M, Jouvion G, Briand D, Thépenier C, et al. Comparative study of injury models for studying muscle regeneration in mice. Plos One. 2016;11(1):e0147198.

40. Mdzomba JB, Joly S, Rodriguez L, Dirani A, Lassiaz P, Behar-Cohen F, et al. Nogo-A-targeting antibody promotes visual recovery and inhibits neuroinflammation after retinal injury. Cell Death Dis. 2020;11(2):1-16.

41. Allagnat F, Fukaya M, Nogueira TC, Delaroche D, Welsh N, Marselli L, et al. C/EBP homologous protein contributes to cytokine-induced pro-inflammatory responses and apoptosis in $\beta$-cells. Cell 
Death Differ. 2012;19(11):1836-46.

42. Kim SH, Kwon D-y, Kwak J-H, Lee S, Lee Y-H, Yun J, et al. Tunicamycin-induced ER stress is accompanied with oxidative stress via abrogation of sulfur amino acids metabolism in the liver. Int $\mathrm{J}$ Mol Sci. 2018;19(12):4114.

43. Nishitoh H. CHOP is a multifunctional transcription factor in the ER stress response. The J Biochem. 2012;151(3):217-9.

44. Jia LX, Zhang WM, Zhang HJ, Li TT, Wang YL, Qin YW, et al. Mechanical stretch-induced endoplasmic reticulum stress, apoptosis and inflammation contribute to thoracic aortic aneurysm and dissection. J Pathol. 2015;236(3):373-83.

45. Zhong, J. et al. Effects of Nogo-A silencing on TNF-a and IL-6 secretion and TH downregulation in lipopolysaccharide-stimulated PC12 cells. Biomed Res Int. 2015 (2015).

46. Egami Y, Fujii M, Kawai K, Ishikawa Y, Fukuda M, Araki N. Activation-inactivation cycling of Rab35 and ARF6 is required for phagocytosis of zymosan in RAW264 macrophages. J Immunol Res. $2015 ; 2015$.

47. Stenina M, Krivov L, Voevodin D, Yarygin V. Phenotypic differences between mdx black mice and mdx albino mice. Comparison of cytokine levels in the blood. Bull Exp Biol Med. 2013;155(3):376-9.

48. Pelosi L, Berardinelli MG, Forcina L, Spelta E, Rizzuto E, Nicoletti C, et al. Increased levels of interleukin-6 exacerbate the dystrophic phenotype in mdx mice. Hum Mol Genet. 2015;24(21):604153.

49. Kharraz Y, Guerra J, Mann CJ, Serrano AL, Muñoz-Cánoves P. Macrophage plasticity and the role of inflammation in skeletal muscle repair. Mediators Inflamm. 2013;2013.

50. Zagrebelsky M, Korte M. Maintaining stable memory engrams: new roles for Nogo-A in the CNS. Neurosci. 2014;283:17-25.

51. Deldicque L. Endoplasmic reticulum stress in human skeletal muscle: any contribution to sarcopenia? Front Physiol. 2013;4:236.

52. Strittmatter SM. Nogo limits neural plasticity and recovery from injury Martin E Schwab and Stephen M Strittmatter 2. Curr Opi Neurobiol. 2014;27:53-60.

53. Masliah E, Xie F, Dayan S, Rockenstein E, Mante M, Adame A, et al. Genetic deletion of Nogo/Rtn4 ameliorates behavioral and neuropathological outcomes in amyloid precursor protein transgenic mice. Neurosci. 2010;169(1):488-94.

54. Schwab DE, Lepski G, Borchers C, Trautmann K, Paulsen F, Schittenhelm J. Immunohistochemical comparative analysis of GFAP, MAP-2, NOGO-A, OLIG-2 and WT-1 expression in WHO 2016 classified neuroepithelial tumours and their prognostic value. Pathol Res Pract. 2018;214(1):15-24.

55. Seiler S, Di Santo S, Widmer HR. Non-canonical actions of Nogo-A and its receptors. Biochem Pharmacol. 2016;100:28-39.

56. Wan J, Benkdane M, Teixeira-Clerc F, Bonnafous S, Louvet A, Lafdil F, et al. M2 Kupffer cells promote M1 Kupffer cell apoptosis: a protective mechanism against alcoholic and nonalcoholic fatty liver 
disease. Hepatol. 2014;59(1):130-42.

57. Cruceriu D, Baldasici O, Balacescu O, Berindan-Neagoe I. The dual role of tumor necrosis factor-alpha (TNF-a) in breast cancer: molecular insights and therapeutic approaches. Cell Oncol. 2020:1-18.

58. Taniguchi K, Karin M. NF-KB, inflammation, immunity and cancer: coming of age. Nat Rev Immunol. 2018;18(5):309-24.

59. Voronov E, Apte RN. IL-1 in colon inflammation, colon carcinogenesis and invasiveness of colon cancer. Cancer microenvironment. 2015;8(3):187-200.

60. Guo M, Härtlova A, Gierliński M, Prescott A, Castellvi J, Losa JH, et al. Triggering MSR1 promotes JNK-mediated inflammation in IL-4-activated macrophages. EMBO J. 2019;38(11).

61. Martinez-Pomares L, Gordon S. Macrophages and Autoimmunity. Autoimmune Dis. Elsevier; 2020. p. 191-212.

62. Martinez FO, Helming L, Gordon S. Alternative activation of macrophages: an immunologic functional perspective. Annu Rev Immunol. 2009;27:451-83.

63. Brozzi F, Nardelli TR, Lopes M, Millard I, Barthson J, Igoillo-Esteve M, et al. Cytokines induce endoplasmic reticulum stress in human, rat and mouse beta cells via different mechanisms. Diabetologia. 2015;58(10):2307-16.

64. Hattori T, Ohoka N, Hayashi H, Onozaki K. C/EBP homologous protein (CHOP) up-regulates IL-6 transcription by trapping negative regulating NF-IL6 isoform. FEBS Lett. 2003;541(1-3):33-9.

65. Choi K, Komurov K, Fletcher JS, Jousma E, Cancelas JA, Wu J, et al. An inflammatory gene signature distinguishes neurofibroma Schwann cells and macrophages from cells in the normal peripheral nervous system. Sci Rep. 2017;7:43315.

66. Lu A, Guo P, Wang L, Tseng C, Huard M, Allen C, et al. Heterogenetic parabiosis between healthy and dystrophic mice improve the histopathology in muscular dystrophy. Sci Rep. 2020;10(1):1-12.

67. Sung S-E, Hwang M, Kim A-Y, Lee E-M, Lee E-J, Hwang S-K, et al. Myod overexpressed equine adipose-derived stem cells enhanced myogenic differentiation potential. Cell Transplant. 2016;25(11):2017-26.

68. Lee E-M, Kim D-Y, Kim A-Y, Lee E-J, Kim S-H, Lee M-M, et al. Chronic effects of losartan on the muscles and the serologic profiles of mdx mice. Life Sci. 2015;143:35-42.

69. Chen X, El Gazzar M, Yoza BK, McCall CE. The NF-kB factor RelB and histone H3 lysine methyltransferase G9a directly interact to generate epigenetic silencing in endotoxin tolerance. J Biol Chem. 2009;284(41):27857-65.

70. Horibata Y, Mitsuhashi S, Shimizu H, Maejima S, Sakamoto H, Aoyama C, et al. The phosphatidylcholine transfer protein StarD7 is important for myogenic differentiation in mouse myoblast C2C12 cells and human primary skeletal myoblasts. Sci Rep. 2020;10(1):1-14.

71. Hong E-G, Ko HJ, Cho Y-R, Kim H-J, Ma Z, Tim YY, et al. Interleukin-10 prevents diet-induced insulin resistance by attenuating macrophage and cytokine response in skeletal muscle. Diabetes. 2009;58(11):2525-35. 
72. Kim D-K, Ryu D, Koh M, Lee M-W, Lim D, Kim M-J, et al. Orphan nuclear receptor estrogen-related receptor Y (ERRY) is key regulator of hepatic gluconeogenesis. J Biol Chem. 2012;287(26):21628-39.

73. Yan X, Huang G, Liu Q, Zheng J, Chen H, Huang Q, et al. Withaferin A protects against spinal cord injury by inhibiting apoptosis and inflammation in mice. Pharm Biol. 2017;55(1):1171-6.

74. Zhou L, Wang L, Lu L, Jiang P, Sun H, Wang H. A novel target of microRNA-29, Ring1 and YY1-binding protein (Rybp), negatively regulates skeletal myogenesis. J Biol Chem. 2012;287(30):25255-65.

75. Bertan F, Wischhof L, Sosulina L, Mittag M, Dalügge D, Fornarelli A, et al. Loss of Ryanodine Receptor 2 impairs neuronal activity-dependent remodeling of dendritic spines and triggers compensatory neuronal hyperexcitability. Cell Death Differ. 2020:1-20.

76. Chen W, Wang W, Sun X, Xie S, Xu X, Liu M, et al. NudCL2 regulates cell migration by stabilizing both myosin-9 and LIS1 with Hsp90. Cell Death Dis. 2020;11(7):1-15.

\section{Figures}

Fig. 1

a

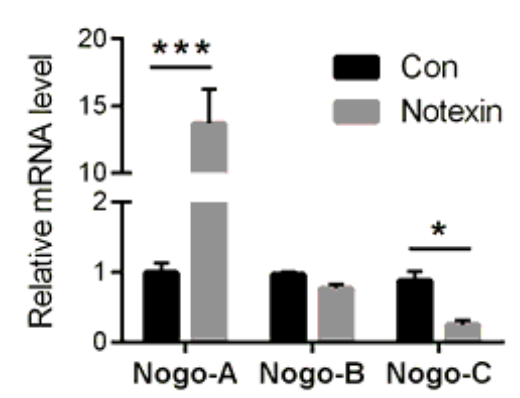

c

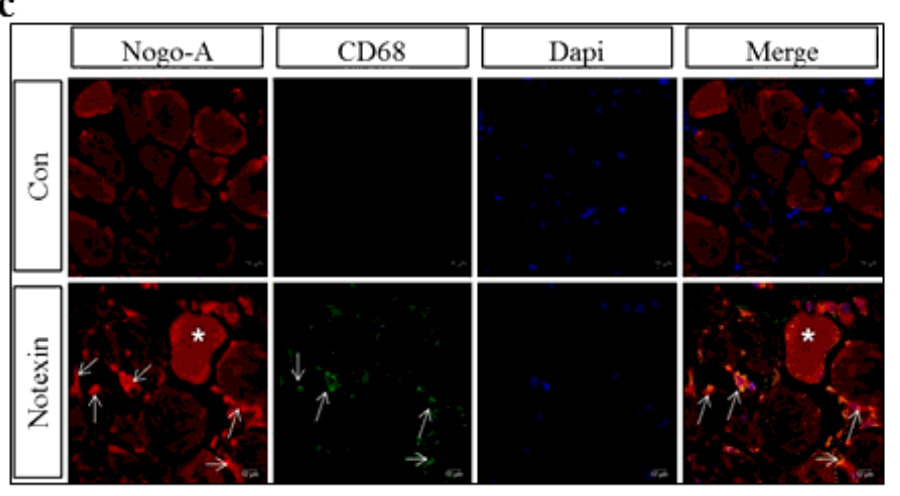

b

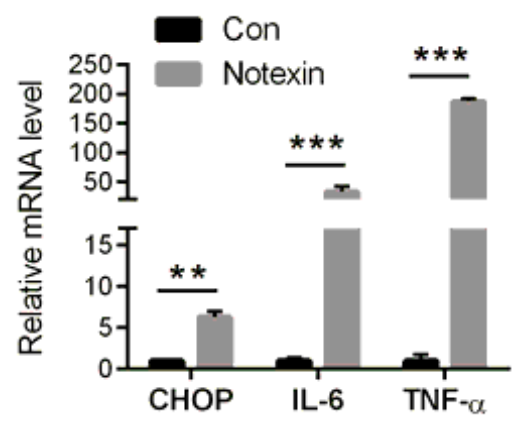

d

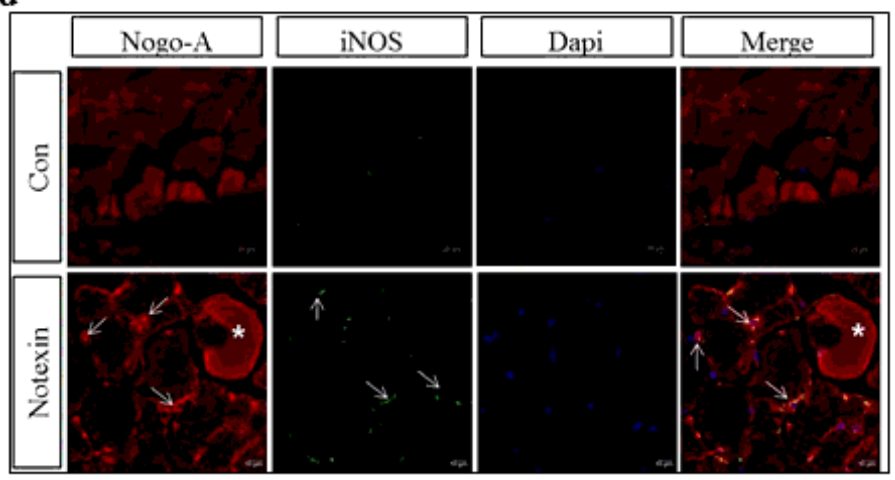


Expression of Nogo-A, CHOP, and pro-inflammatory factors is increased in muscle from notexin-treated mice. a Nogo-A is upregulated in injured muscle, while levels of Nogo-B and $C$ are not significantly altered. Notexin-injured muscle expresses higher levels of Nogo-A compared with those in the WT control $(n=3)$. $b$ Expression of Nogo-A, CHOP, IL-6, and TNF- $a$ is upregulated in notexin-injured muscle $(n=3)$. c Notexininjured muscle was immunostained using antibodies against Nogo-A and CD68 $(n=3)$. Nogo-A is localized to muscle fibers (asterisk) and colocalizes with CD68 (arrow). $d$ Notexin-injured muscle was immunopositive for Nogo-A and iNOS ( $n=3)$. Nogo-A is localized to muscle fibers (asterisk) and colocalizes with iNOS (arrow). 3 days after a single intramuscular injection of notexin $(12.5 \mu \mathrm{g} / \mathrm{ml}, 20 \mu \mathrm{l}$

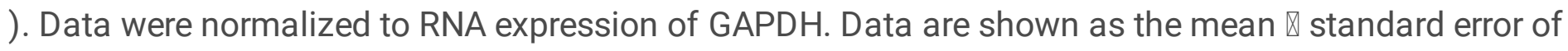
the mean. The statistical significance was determined using Student's t-test. Data are denoted by asterisks, where ${ }^{*} P<0.05,{ }^{\star *} P<0.01$, and ${ }^{\star * \star} P<0.001$. Secondary antibodies used were Alexa Fluor (AF)-555 and AF-488. Scale bar, $10 \mu \mathrm{m}, \times 400$ magnification.

Fig. 2

a

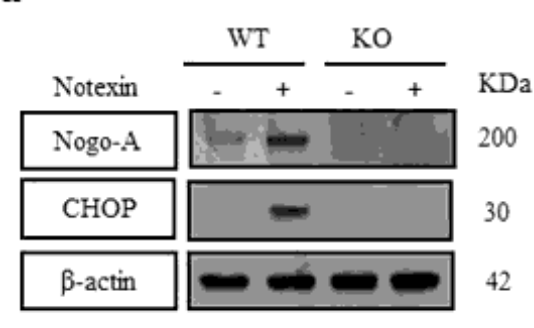

b

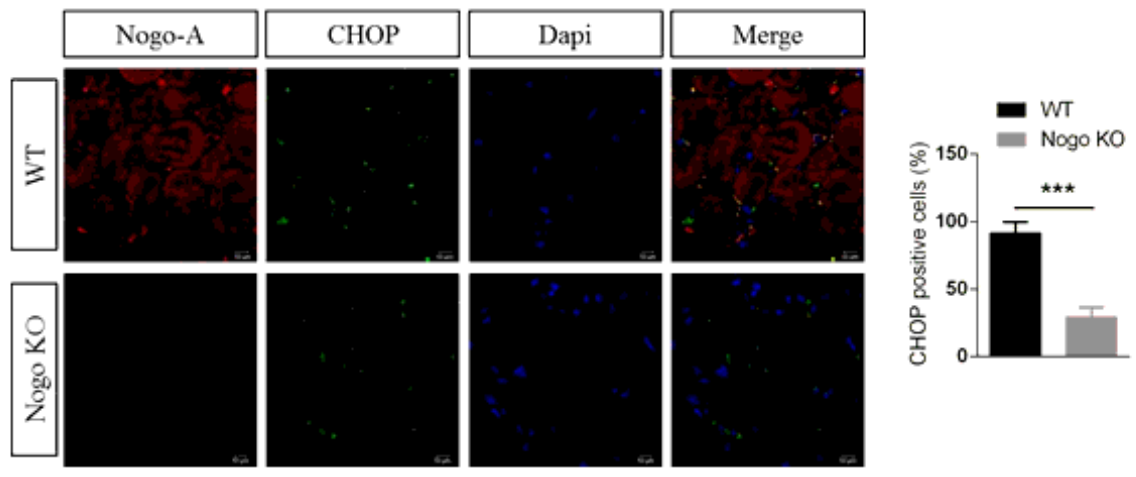

e

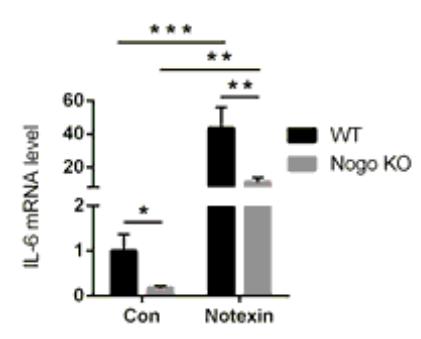

$\mathbf{f}$

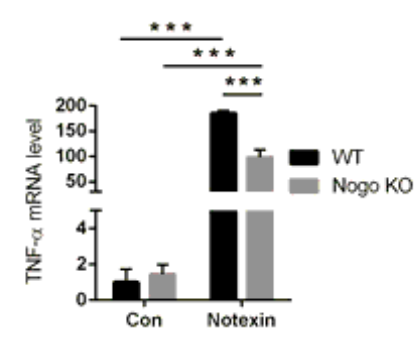

\section{Figure 2}

Pro-inflammatory factors mediated by $\mathrm{CHOP}$ signaling. a Nogo-A, CHOP, and $\beta$-actin protein expression levels in muscle from notexin-treated WT mice compared with muscle from notexin-treated Nogo-KO mice 
$(n=3)$. b Expression of CHOP in injured muscle from WT mice compared with its expression in injured muscle from Nogo-KO mice ( $n=3$ ) by immunofluorescence (IF). c-f Nogo-A, CHOP, IL-6, and TNF-a mRNA expression levels in gastrocnemius muscle isolated from WT $(n=3)$ and Nogo-KO mice $(n=3) 3$ days after a single intramuscular injection of notexin $(12.5 \mu \mathrm{g} / \mathrm{ml})$. Data were normalized to RNA expression of GAPDH. In all, $40 \mu \mathrm{g}$ of protein was used for immunoblot (IB) experiments. Data are shown as the mean $\square$ standard error of the mean. Statistical significance was determined using Student's t-test. Secondary antibodies used were Alexa Fluor (AF)-555 and AF-488. Significant data are denoted by asterisks where ${ }^{*} \mathrm{P}<0.05,{ }^{* * P}<0.01$, and ${ }^{* * *} \mathrm{P}<0.001$. Scale bar, $10 \mu \mathrm{m}, \times 400$ magnification.

Fig. 3
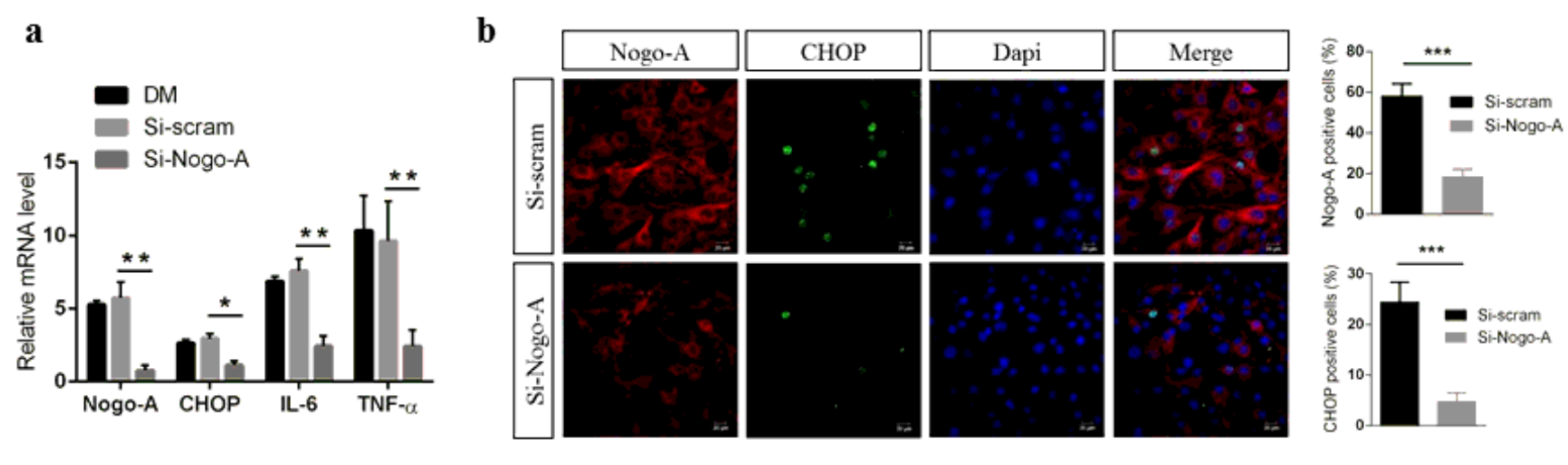

c

d
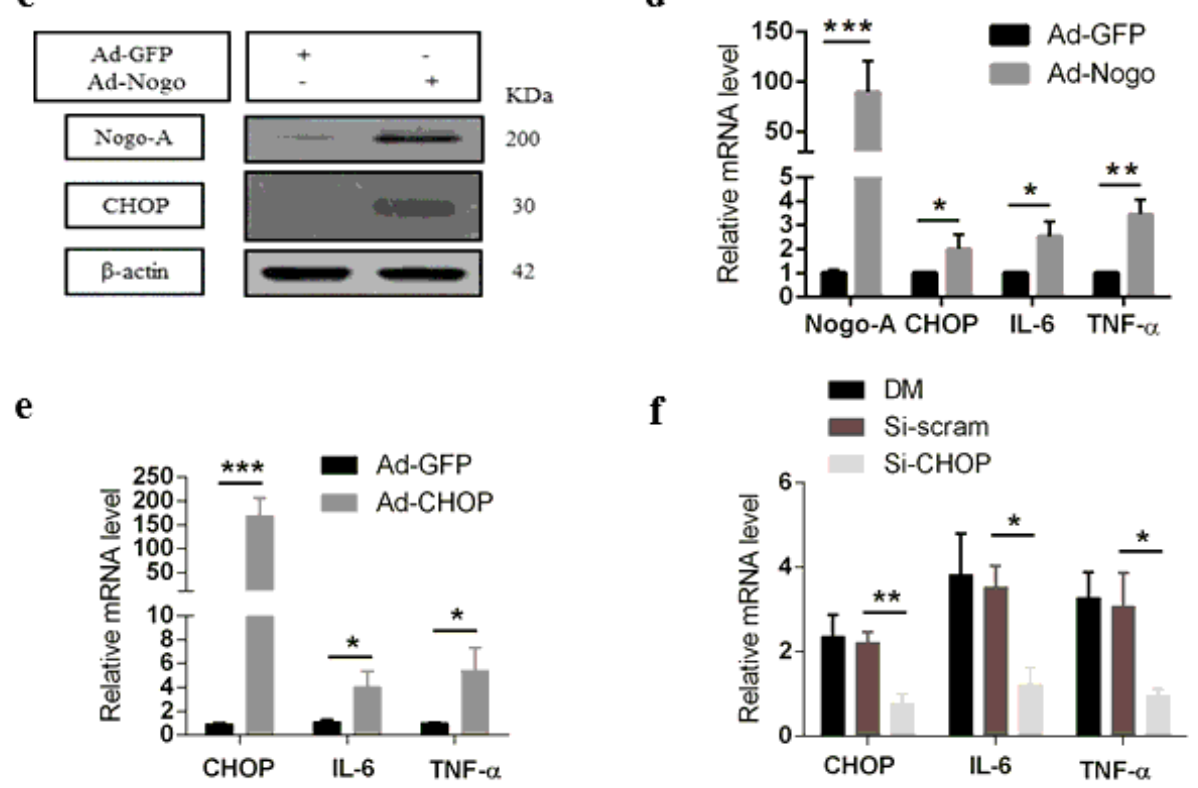

\section{Figure 3}

Nogo-A enhances CHOP expression and production of pro-inflammatory factors in $\mathrm{C} 2 \mathrm{C} 12$ cells. a C2C12 cells were transfected with si-Nogo-A or si-scramble, after which the cells were differentiated (DM) for 3 days and then harvested for qPCR analysis of Nogo-A, CHOP, IL-6, and TNF-a expression levels. b Nogo-A and $\mathrm{CHOP}$ antibodies were used to stain transfected $\mathrm{C} 2 \mathrm{C} 12$ cells for IF analysis. c Cell extracts were analyzed by immunoblot (IB) analysis for Nogo-A, CHOP, and $\beta$-actin expression. $d$ C2C12 cells were infected with Ad-GFP and Ad-Nogo-A (60 MOI) for 24 h. Nogo-A, CHOP, IL-6, and TNF-a mRNA levels were 
analyzed by qPCR. e Ad-GFP and Ad-CHOP (60 MOI) were used to infect C2C12 cells for $24 \mathrm{~h}$. qPCR analysis of CHOP, IL-6, and TNF-a expression. $f$ C2C12 cells were transfected with si-CHOP or si-control. After transfection for $36 \mathrm{~h}$, the cells were differentiated for 3 days and then qPCR was performed to determine CHOP, IL-6, and TNF-a expression. In all, $40 \mu \mathrm{g}$ of protein was used for IB. Data are shown as the mean $\otimes$ standard error of the mean. Statistical significance was determined using Student's t-test. The $\beta$-actin level was used for normalization of the expression levels. Data are denoted by asterisks where *P $<0.05,{ }^{\star} \mathrm{P}<0.01,{ }^{\star * \star} \mathrm{P}<0.001$. Alexa Fluor $(\mathrm{AF})-488$ and AF-555 were used as secondary antibodies.

Scale bar, $10 \mu \mathrm{m}, \times 400$ magnification.

Fig. 4

a

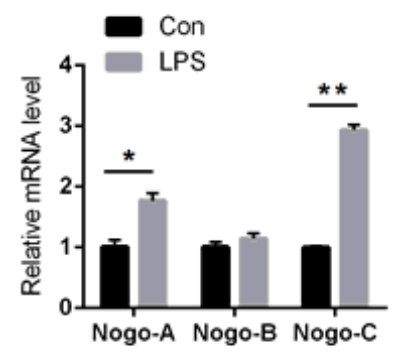

c

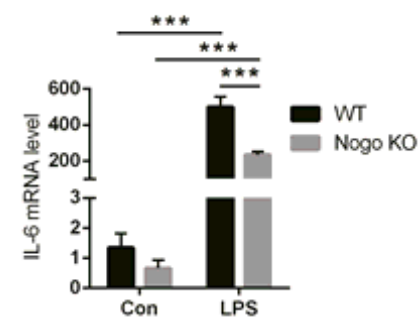

g

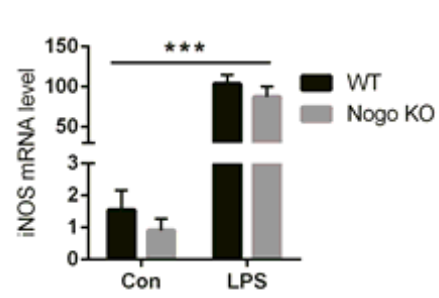

b

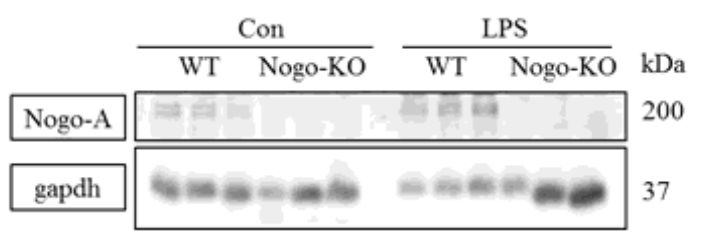

e

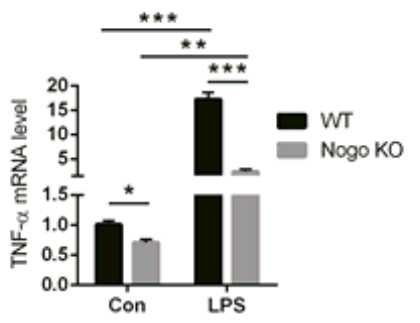

h

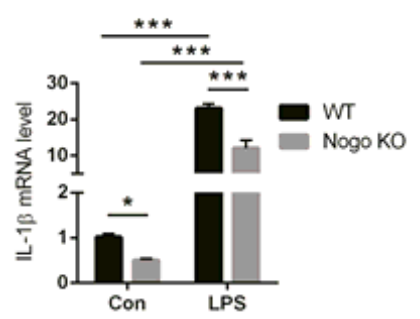

i
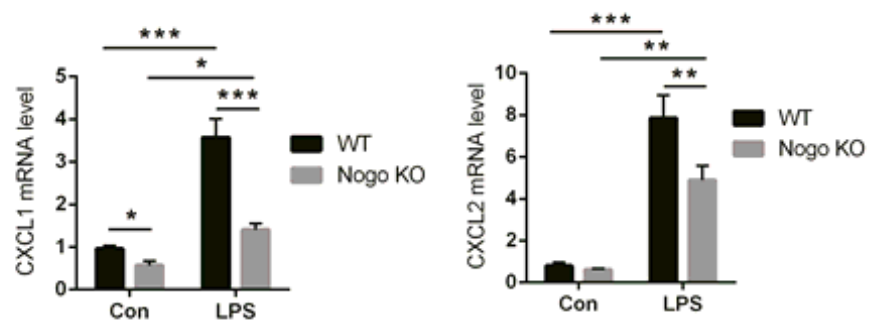

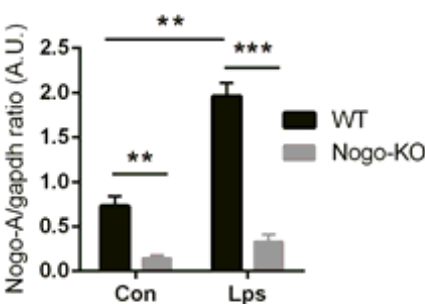

f

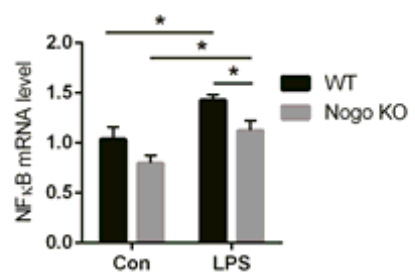

\section{Figure 4}

Nogo deficiency inhibits pro-inflammatory gene expression in BMDM after LPS treatment. a Nogo-A and $\mathrm{C}$, but not Nogo-B, are upregulated in LPS-treated bone marrow- derived macrophages (BMDM) compared with the levels in control BMDM $(n=3)$. b IB data of control and LPS-treated BMDM. Nogo-A was increased in response to LPS treatment in WT BMDM compared with the levels in Nogo-KO BMDM $(n=$ 3). $c$-i mRNA levels of pro-inflammatory factors including IL-6, TNF-a, IL-1 $\beta, N F-K B$, iNOS, CXCL1, and CXCL2 in control and LPS-treated WT and Nogo-KO BMDM $(n=4)$. In all, $30 \mu \mathrm{g}$ of protein was used in IB. 
GAPDH was used for normalization. Data are shown as the mean $\otimes$ standard error of the mean. Statistical significance was determined using Student's t-test. Data are denoted by asterisks, where *P $<0.05, * \star P<$ 0.01 , and $* * * P<0.001$.

Fig. 5

$\mathbf{a}$

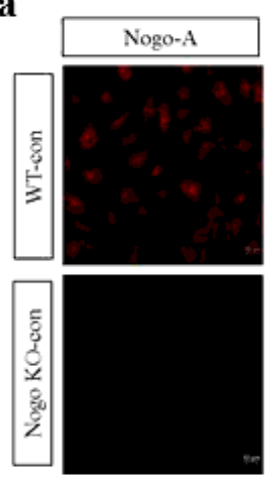

c

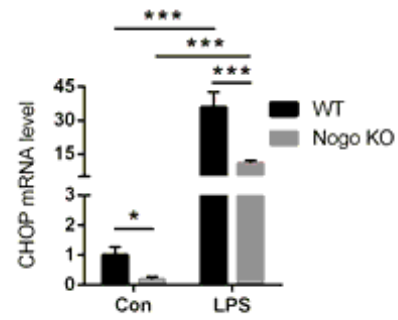

$\mathbf{f}$

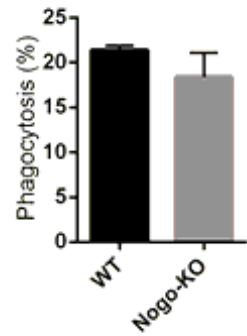

b
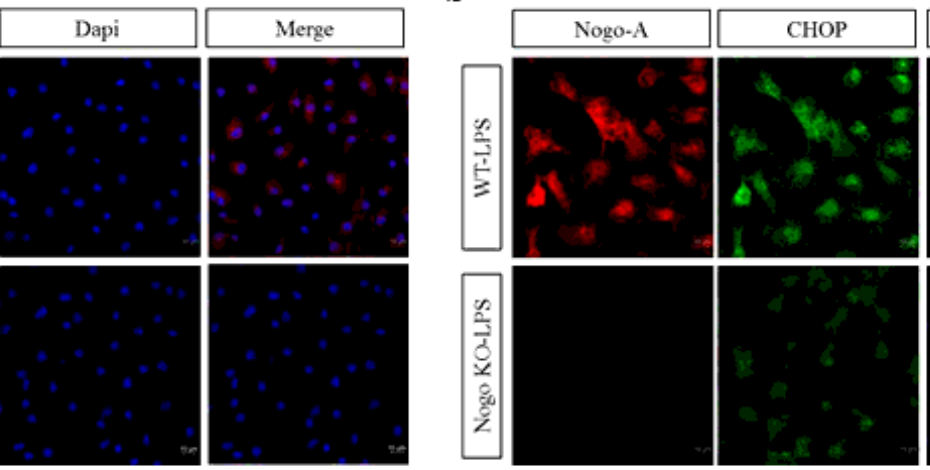

d

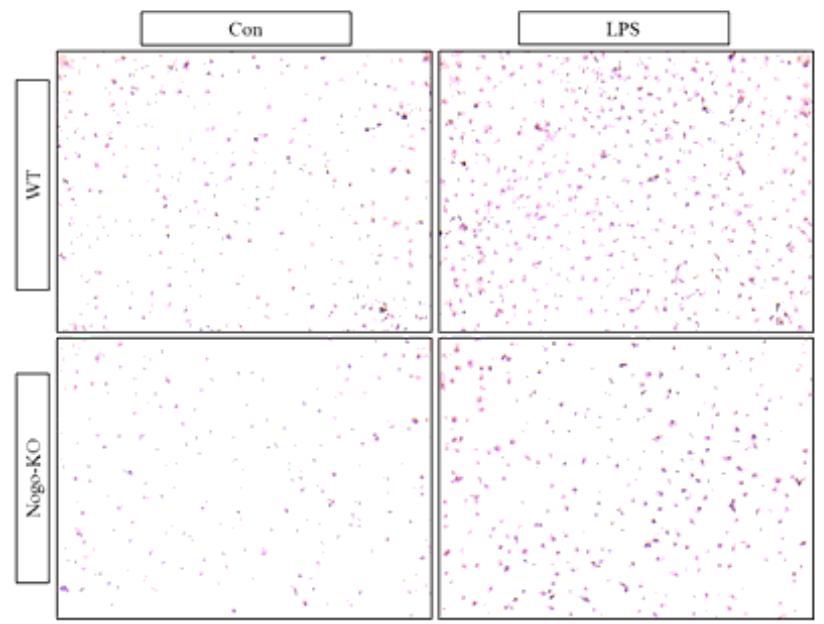

e

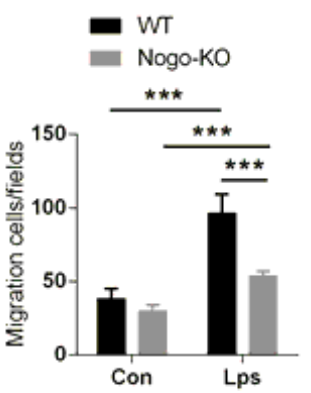

\section{Figure 5}

Nogo deficiency inhibits CHOP signaling and migration of BMDM after LPS treatment. a IF results of Nogo-A and CHOP in control WT BMDM and Nogo-KO BMDM ( $\mathrm{n}=3)$. Nogo-A is expressed in control WT BMDM, while CHOP expression is absent in control WT and Nogo-KO BMDM. b IF results show that NogoA (red) and CHOP (green) are increased in LPS-treated WT BMDM compared with the levels in Nogo-KO BMDM. c Level of CHOP mRNA is upregulated in LPS-treated WT BMDM compared with LPS-treated Nogo-KO BMDM $(n=4)$. d Migration assay using WT and Nogo-KO BMDM $(n=3)$. Nogo-KO BMDM exhibit a lower migration ability compared with WT BMDM after LPS treatment (100 ng/ml) for $24 \mathrm{~h}$. e Quantification of BMDM migration reveals a significantly lower migration ability in LPS-treated Nogo-KO MMDM compared with WT BMDM $(n=3)$. No significant difference was observed in migration activity between control WT and Nogo-KO BMDM. f Phagocytosis by macrophages from WT and Nogo-KO BMDM after treatment with fluorescent bioparticles of the pro-inflammatory cytokine inducer zymosan ( $\mathrm{n}$ 
= 3). The numbers of phagocytes were analyzed by flow cytometry. Data are shown as the mean $\square$ standard error of the mean. Statistical significance was determined using Student's t-test. Data are denoted by asterisks, where ${ }^{*} P<0.05,{ }^{*} \mathrm{P}<0.01$, and ${ }^{* \star *} \mathrm{P}<0.001$. Alexa Fluor (AF)-555 and AF-488 were used as secondary antibodies. Scale bar, $10 \mu \mathrm{m}, 400 x$ magnification.

\section{Fig. 6}

a

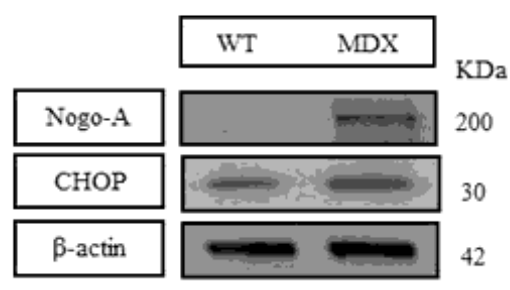

c

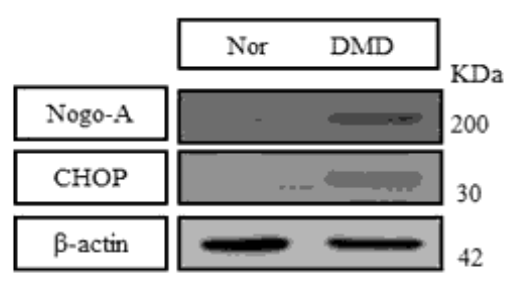

b

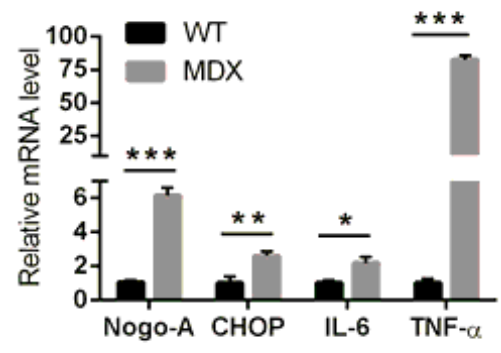

e

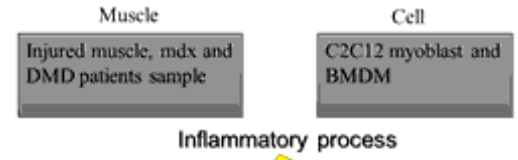

d

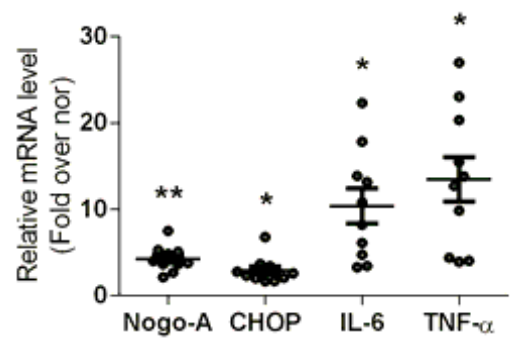

Inflammatory process

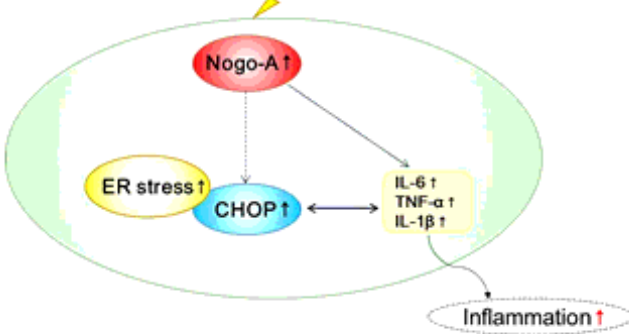

Figure 6

Nogo-A, $\mathrm{CHOP}$, and pro-inflammatory factors are upregulated in $\mathrm{mdx}$ mice and DMD patient samples. a IB analysis of Nogo-A, CHOP, and $\beta$-actin in the skeletal muscle of WT and mdx mice. b qPCR analysis of mRNA from the skeletal muscle of 12-week-old WT and mdx mice $(n=4)$. c Tissue extracts from DMD patients were used in the IB analysis with Nogo-A, CHOP, and $\beta$-actin antibodies. $d$ qPCR analyses were performed on biopsy samples of DMD patients $(n=10)$ and normal patients $(n=5)$. e Proposed model of the role of Nogo-A in the regulation of inflammation. Nogo-A is activated in muscle from notexin-treated mice, mdx mice, DMD patients and in LPS-treated BMDM. Subsequently, Nogo-A expression may be accompanied by CHOP activation and can also activate pro-inflammatory cytokines and chemokines in injured or degenerated muscle and in LPS-stimulated BMDM. We conclude that Nogo-A exerts inflammatory effects. In all, $40 \mu \mathrm{g}$ of protein was used for the IB experiments. The statistical significance 
was determined using Student's t-test. Error bars represent the standard error of the mean. Data are denoted by asterisks where ${ }^{*} P<0.05, * \star P<0.01$, and ${ }^{\star * *} P<0.001$.

\section{Supplementary Files}

This is a list of supplementary files associated with this preprint. Click to download.

- SupplFigureLegendsCellBioscienceJKS.docx

- Fig.S1.TIF

- Fig.S2.TIF

- Fig.S3.TIF

- Fig.S4.TIF

- Fig.S5.TIF

- Fig.S6.TIF

- TableS1CellBioscienceJKS.pdf 